\title{
Direct Electron Beam Writing of Silver-based
}

\section{Nanostructures}

Katja Höflich ${ }^{* 1,2}$, Jakub Jurczyk ${ }^{1,3}$, Yucheng Zhang ${ }^{1}$, Marcos V. Puydinger dos Santos ${ }^{1,4}$,

Maximilian Götz ${ }^{2}$, Carlos Guerra-Nuñez ${ }^{l}$, James P. Best ${ }^{l}$, Czeslaw Kapusta ${ }^{3}$, and Ivo Utke

1 Empa - Swiss Federal Laboratories for Materials Science and Technology, Laboratory for

Mechanics of Materials and Nanostructures, Feuerwerkerstrasse 39, CH - 3602 Thun,

Switzerland

2 Helmholtz-Zentrum Berlin für Materialien und Energie, Nanoscale Structures and Microscopic Analysis, Hahn-Meitner-Platz 1, D - 14109 Berlin, Germany

3 AGH University of Science and Technology Krakow, Faculty of Physics and Applied Computer Science, Al. Mickiewicza 30, 30-059 Kraków, Poland

4 Institute of Physics Gleb Wataghin, University of Campinas, Rua Sergio Buarque de Holanda 777 Cidade Universitaria, 13083-859 Campinas-SP, Brazil

KEYWORDS: electron beam induced deposition, direct writing, silver, nanostructures, precursor compounds 


\section{ABSTRACT}

Direct writing utilizing a focused electron beam constitutes an interesting alternative to resist based techniques, as it allows for precise and flexible growth onto any conductive substrate in a singlestep process. One important challenge, however, is the identification of appropriate precursors which allow for deposition of the material of choice e.g. for envisaged applications in nano-optics. In this regard the coinage metal silver is of particular interest since it shows a relatively high plasma frequency, and thus, excellent plasmonic properties in the visible range. By utilizing the precursor compound $\mathrm{AgO}_{2} \mathrm{Me}_{2} \mathrm{Bu}$, direct writing of silver-based nanostructures via local electron beam induced deposition could be realized for the first time. Interestingly, the silver deposition was strongly dependent on electron dose; at low doses of $30 \mathrm{nC} / \mu \mathrm{m}^{2}$ a dominant formation of pure silver crystals was observed, while at higher electron doses around $10^{4} \mathrm{nC} / \mu \mathrm{m}^{2}$ large carbon contents were measured. A scheme for the enhanced silver deposition under low electron fluxes by an electronic activation of precursor dissociation below thermal CVD temperature is proposed and validated using material characterization techniques. Finally, the knowledge gained was employed to fabricate well-defined two-dimensional deposits with maximized silver content approaching 75 at.\%, which was achieved by proper adjustment of the deposition parameters. The corresponding deposits consist of plasmonically active silver crystallites and demonstrate a pronounced Raman signal enhancement of the carbonaceous matrix. 


\section{INTRODUCTION}

Employing electrons for direct writing is highly desirable since it provides both, the highest resolution and three-dimensional flexibility ${ }^{1,2}$. The corresponding technique, termed electron beam induced deposition (EBID) utilizes focused electrons for the dissociation of molecular precursors which are locally introduced into the vacuum chamber of an electron microscope $\mathrm{e}^{3,4}$. Compared to standard multistep lithographic techniques for nanostructure fabrication, EBID stands out as a mask-less single-step approach. Typically, metal organic precursor media are employed to finally realize metallic nanostructures ${ }^{5}$. In an ideal case the electron-beam induced dissociation would separate precursor molecules adsorbed onto the substrate into their volatile organic ligands (which subsequently are pumped out) and the central metal atoms forming the deposit. However, a substantial amount of carbon from the co-dissociated ligands is generally incorporated into the deposit since primary, back-scattered and generated secondary electrons involved in the dissociation process cover a wide energy range, which is not selective to bond energies ${ }^{6,7}$. Typical metal contents for EBID from state-of-the-art metal-organic precursors range from about 5 at.\% to 40 at.\% including $\mathrm{Au}, \mathrm{Cu}, \mathrm{Pt}, \mathrm{W}, \mathrm{Mo}, \mathrm{Cr}, \mathrm{Si}, \mathrm{Ti}$, and $\mathrm{Ni}^{3,5}$. Hence, the challenges of EBID lie in depositing the material of choice in sufficient quality for the envisaged applications, e.g. in plasmonics $^{8-10}$. In that respect, post-growth purification mainly relying on oxidation of the codeposited carbonaceous matrix constitutes an important field of research ${ }^{5}$, providing promising results for already established precursor compounds. Recent examples include room-temperature methods like electron beam curing ${ }^{11,1213}$ or oxygen plasma treatment ${ }^{14}$, as well as laser-assisted purification $^{15}$ and heating in different atmospheres ${ }^{16,17}$ or vacuum ${ }^{18}$.

To avoid carbon co-deposition from the start one can rely on purely inorganic precursor compounds $^{19}$, low carbon-content precursors ${ }^{20,21}$ or employ the catalytic activity of the precursors 
by using electrons as an invisible ink prior to deposition ${ }^{22}$. A further important approach includes the identification and/or design of novel precursor compounds ${ }^{23}$, to achieve both a broadening of the available materials to be deposited by EBID with a preferably low or no carbon co-deposition.

The choice of appropriate precursors usually starts from compounds successfully proven for chemical deposition techniques and involves several steps. Once a potentially interesting low vapor pressure compound is identified by bulk exposure ${ }^{20,24}$, the following challenging tasks must be solved: (I) supply of the vapor phase to the substrate and to location of electron exposure, (II) physisorption onto the surface - preferentially without condensation to keep the 3D writing capability of EBID, although sub-micron condensation may also be suitable in some cases ${ }^{24-26}$, and (III) desorption of intact adsorbates wherever not exposed to electrons. By utilizing this approach novel inorganic gold precursors have been identified ${ }^{20}$. More recently, a cisplatinum precursor was investigated by bulk exposure vs. condensed monolayer exposure ${ }^{24}$ demonstrating the validity as well as peculiarities of such an approach.

Although today a wealth of metals are accessible using electron beam induced deposition ${ }^{5}$, no silver-based EBID process has been reported yet. Up to now only liquid phase silver EBID ${ }^{27-29}$ was realized accompanied by the typical severe restrictions in resolution and flexibility. Since silver is ideally suited for plasmonic applications due to its small optical losses in the whole visible regime, there is an urgent need for the development of high-precision direct writing of silver nanostructures.

To address this challenging task, the compound Ag-(2,2-dimethylbutanoato- $\mathrm{KO})-\left(\mathrm{AgO}_{2} \mathrm{Me} 2 \mathrm{Bu}\right)$ was identified as most promising. The carboxylate $\mathrm{AgO}_{2} \mathrm{Me}_{2} \mathrm{Bu}$ is known for chemical vapor deposition (CVD) $)^{30,31}$ and, as it turned out, affords an extraordinary sensitivity to electron beam 
impact. While this is highly desirable for the direct writing of nanostructures with a high metal content, the associated experimental conditions are extremely demanding. To account for silver deposition by electron beam impact, a fully integrated gas injection system had to be developed, assembled and subsequently optimized regarding chemical inertness and short molecule paths. Thereby, gas-phase electron beam induced deposition of silver could be demonstrated for the first time.

\section{RESULTS AND DISCUSSION}

Figure 1 (a) and (b) show exemplary scanning electron micrographs of typical spot deposits from $\mathrm{AgO}_{2} \mathrm{Me}_{2} \mathrm{Bu}$ on different substrates. The temperature of the substrate was kept constant at around $120^{\circ} \mathrm{C}$, while the evaporation temperature of the gas injection system (GIS) was set to $150^{\circ} \mathrm{C}$. EBID was performed by irradiating stationary spot exposure for 5 minutes. As a consequence of the GIS design, the gas flux was constant during deposition and in-situ imaging. A $15 \mathrm{kV}$ acceleration voltage and beam current of $0.5 \mathrm{nA}$ onto silicon results in a local electron dose of around $3 \times 10^{3} \mathrm{nC} / \mu \mathrm{m}^{2}$ within the full width at half maximum of around $250 \mathrm{~nm}$ of the primary electron beam.

As visible in Figure 1 (a) spot deposits onto silicon are surrounded by a halo with a diameter of around $4.5 \mu \mathrm{m}$. The diffuse shapes of the deposits onto silicon correspond strongly to the radius of backscattered electrons and their secondary electrons. In Figure 1(b) spot deposits with a reduced current of $0.15 \mathrm{nA}$ on a roughly $20 \mathrm{~nm}$ thick carbon membrane of a TEM grid are shown. The membrane deposits indicate the impinging primary electron beam profile to a width of around $500 \mathrm{~nm}$ in diameter and lack the halo region as there is no bulk interaction volume. 
Figure 1 (c) displays the radial density distributions of backscattered (BSE) and generated secondary electrons (SE) obtained from Monte-Carlo modeling for a silicon substrate (red curve) and the carbon membrane (blue curve). The convolution was performed with the Gaussian shape of the primary beams and their respective FWHM (250 nm for $0.5 \mathrm{nA}$ and $160 \mathrm{~nm}$ for $0.15 \mathrm{nA}$ ) as determined in resolution tests (see Supporting Information, Supp4). For the silicon substrate the density of secondary and backscattered electrons decreases by almost two orders of magnitude within the first $250 \mathrm{~nm}$ of radial distance following the Gaussian primary beam profile. Outside the focal region, for radii $>250 \mathrm{~nm}$, the BSE and SE profile is dominated by the interaction volume. At a radius of $2 \mu \mathrm{m}$ it shows a value of about $10^{-7} \mathrm{e}^{-} / \mathrm{nm}^{2}$, whereby it starts sharply decreasing and approaching zero at around $2.25 \mu \mathrm{m}$. This corresponds well to the observed radius of the halo around the spot deposits on bulk silicon. At $2 \mu \mathrm{m}$ distance from the beam center the electron dose amounts to ca. $30 \mathrm{nC} / \mu \mathrm{m}^{2}$ at maximum in these peripheral halo regions compared to $3 \times 10^{3} \mathrm{nC} / \mu \mathrm{m}^{2}$ in the focal region. According to this significantly decreased number of available electrons in the peripheral region a $100-10000 x$ lower deposition rate compared to that in the beam center would be expected for the case of an electron-limited growth regime. However, in the focal region the deposition is strongly adsorbate-limited due to the low vapor pressure of the compound (see Supporting Information, Supp5). In the beam center the number of electrons is about three to four orders of magnitude larger than the number of impinging precursor molecules, thus severely limiting the deposition rate. Interestingly, a particular ring pattern is observed exhibiting features which suggest the formation of silver crystals. This ring region became even more pronounced when observing the spot deposit with continuing precursor flux in SEM observation as routinely done for documentation purposes. Experiments with continued gas flow without electron beam 
irradiation showed no further silver growth (see Supporting Information, Supp9). Hence, this effect can be attributed to a post-growth EBID under low electron fluxes as present in SEM scanning.

Figures 1 (d) and (e) propose a model scheme of the underlying processes: After physisorption onto the substrate, $\mathrm{AgO}_{2} \mathrm{Me}_{2} \mathrm{Bu}$ molecules are dissociated into silver and ligands by electron impact. Under high electron flux the precursor ligands are co-dissociated resulting in a microstructure of metal nanoparticles embedded into a carbonaceous matrix known for metalorganic precursor media. For this regime, and typical for EBID, the occurrence of a large carbon amount as well as oxygen is observed in the deposits. Furthermore, the carbon co-deposition is expected to reduce the rates of adsorption of further molecules and desorption of their ligands and, thus, hinders the formation of larger silver particles. As shown in Figure 1 (e) for low electron flux the deposition process becomes electron-limited and the desorption rate of the ligands becomes larger than their dissociation rate leaving un-poisoned silver crystals. Thus, the forming silver nanocrystals are not embedded into a carbonaceous matrix and can act as preferred adsorption sites giving rise to efficient growth of pure silver. In case of the spot deposit the formation rate of these silver crystals depends on the radial electron flux distribution. As the electron flux quickly decays by orders of magnitude a ring-like silver feature is formed. Beyond that ring, the overall deposition rate is too small for the formation of visibly large nuclei. Yet the dependence of silver deposition on the electron flux is non-linear, as competitive co-deposition of the adsorbate ligand fragment is involved which in turn influences the adsorption and desorption of further molecules and ligand fragments.

While this behavior, especially the small vapor pressure of the compound in combination with its strong chemical activity and the sensitivity even to low energy electrons, might complicate the fabrication of high aspect ratio structures, it provides an ideal platform for the tuning of the 
material composition and, thus, for selective growth of high metal content deposits. Furthermore, using short pulse spot exposure to realize corresponding low doses as prevailing the halo region will enable the same sub-10 $\mathrm{nm}$ spatial resolution as achieved in $\mathrm{EBID}^{32,33}$.

Since the electron flux on the surface varies over several orders of magnitude around the focal region of an electron beam, the whole area can be taken in terms of a combinatorial approach for observing the different deposition regimes. To further study these regimes with respect to composition and to eliminate the influence of the substrate, a massive spot deposit was fabricated with an exposure time of $1 \mathrm{~h}$ resulting in a deposit thickness of $300 \mathrm{~nm}$.

Figure 2 (a) shows the scanning electron micrograph of the corresponding deposit, where different regions of deposition are immediately visible: The darker central region highlights the co-deposition of carbon while the bright spots are pure silver crystals. The overlaid graph visualizes the density of the secondary and backscattered electrons scaled to the spatial extension of the spot deposit. In Figure 2 (b) the high angle annular dark field scanning transmission electron micrograph (HAADF-TEM) taken from a cross-section through the deposit (as marked in Figure 2 (a)) shows the typical metal crystals embedded in the carbonaceous matrix for the EBID regime. In the surface region pure silver growth took place resulting in silver crystals of increasing sizes of up to $100 \mathrm{~nm}$ as visible in Figure 2 (a). The size of the silver crystals on top (see Figure 2 (b) increased by the scanning of the electron beam after the actual spot deposition for imaging and thereby providing continued silver growth in the electron-limited regime, as already discussed for Fig. 1a. The thin-film corrected EDX quantification shown in Figure 2 (d) suggests a silver content up to 70 at. $\%$ for the case of pure silver on top, where below a typical EBID composite is situated. In the neighboring central EBID region, the silver amount is about 42 at.\%, with a carbon content slightly exceeding that of silver. In both cases the amount of metal is remarkable, given that the 
typical metal content of deposits from organo-metallic precursor compounds with a comparable amount of carbon within the ligands is in the order of 5-40 at. $\%$ at maximum ${ }^{5}$.

Concerning the deposition pathways, the small oxygen content in the (background-corrected) stoichiometry of the EBID region $(\mathrm{Ag}: \mathrm{O}: \mathrm{C} \approx 6: 1: 7)$ suggests only a minor contribution of full polymerization of the carboxylate compound to the deposit growth. In parallel, for low secondary electron energies (around $1-50 \mathrm{eV}$ ), dissociative electron attachment and dissociation into neutrals and ions could separate the reduced silver atom from the complete ligand. Subsequently, the ligands either desorb from the substrate or dissociate into species of which the oxygen containing ones desorb while some of the carbon species are co-deposited ${ }^{7}$.

For the case of two-dimensional depositions based on $\mathrm{AgO}_{2} \mathrm{Me}_{2} \mathrm{Bu}$ the larger area and thus smaller local electron flux can be employed for optimal desorption of ligands during the electron beam induced deposition, resulting in a significantly higher silver content than expected for the typical EBID case. For that purpose a 'slow' electron beam movement over areas of $10 \mathrm{x} 10 \mu \mathrm{m}^{2}$ was applied, with a duration of $11 \mathrm{~s}$ for one full scan realized by a dwell time of $1 \mu$ s and a pitch of $20 \mathrm{~nm}$. The area was scanned with a current of around $0.5 \mathrm{nA}$, resulting in an electron dose of $0.05 \mathrm{nC} / \mu \mathrm{m}^{2}$ per full scan. In contrast to the spot deposits, where the beam resides at a fixed position, here an efficient supply and activation of a large number of adsorbates can be achieved. Figure 3 (a) shows a typical planar deposit after 100 passes, where pure silver crystals are formed while still ensuring the selectivity of EBID. As visible from Figure 3 (a) the mean size diameters of the larger particles range from 50 to $400 \mathrm{~nm}$ with an average size of around $115( \pm 20) \mathrm{nm}$ following a log-normal distribution as depicted in the histograms in 3 (b). The deposit consists of a single layer of pure silver crystals (see Supporting Information, Supp8). As mentioned before post-deposition irradiation for imaging resulted in further deposition since the gas flow could not 
be interrupted. Hence, distinctly smaller particles onto the deposit are visible in Figure 3 (a), here with an average size of $7 \mathrm{~nm}$ derived from the size distribution shown in Figure 3 (b). These particles increase in number and size for continued electron beam impact (see Supporting Information Supp8).

Figure 3 (c) presents the results from EDX investigation after thin-film correction for the square in Figure 3 (a). The inset shows the corresponding AFM image from which the layer thickness was extracted as $95( \pm 15) \mathrm{nm}$. For the as-deposited 2D structure, an extremely high silver content of around $73( \pm 2)$ at.\% could be achieved ${ }^{1}$. It is linked to the deposition parameters and does not change after electron beam irradiation fully ceases. As mentioned before, in control experiments with continuous gas flow over several hours without further electron beam irradiation, no further growth is observed (see Supporting Information, Supp9). This provides evidence that the growth of silver is determined by the presence of electrons.

The balance between dissociation and electron-stimulated desorption enables the formation of the pure metal. The presence of silver was proven by thorough evaluation of high-resolution transmission electron microscopy (HRTEM) excluding the occurrence of any silver oxides (see Supporting Information, Supp7). Figure 3 (d) shows a HRTEM image of a crystal with a distance of $0.23 \mathrm{~nm}$ between the (111) lattice planes as expected for silver. In the corresponding fast Fourier transformed (FFT) image displayed in Figure (e), the diffraction spots for silver $\{111\}$ and $\{002\}$ are marked.

To finally demonstrate the potential for plasmonic applications, the surface enhanced Raman scattering (SERS) properties were examined for two-dimensional silver deposits. It was

\footnotetext{
${ }^{1}$ This value was determined using thin-film and background corrections for the carbon background in the EDX measurements of around 15-16 at.\% (see Supporting Information, Supp5).
} 
investigated whether the carbon signal from the residual carbon content could be enhanced in the region of the silver deposits, using a $532 \mathrm{~nm}$ laser source. As shown in Figure 4 (a) a 2D Raman map was performed over the deposit, and the maximum signal intensity for each Raman spectrum was evaluated. The extracted spectra presented in Figure 4 (b) prove the distinct and significant signal enhancement in the deposit region due to plasmonic excitation of the silver particles. The signal enhancement was between 100x - 400x, depending on the nature of the local deposit, which is reasonable when compared to other nanoparticle Ag-based SERS systems with smaller dimensions ${ }^{34}$. The enhanced spectra show both $\mathrm{D}$ and $\mathrm{G}$ band features from the residual carbon matrix, and further highlight the complexity of the spectra in the organic vibration range, most likely due to other carbon-based molecular vibrations from electron induced fragments of precursor molecules. From the enhanced spectra the intensity ratio of $\mathrm{D}$ and $\mathrm{G}$ band were evaluated using a Lorentzian peak fitting, and suggest the formation of nanocrystalline graphite clusters in a disordered carbon matrix ${ }^{35,36}$. The obtained average carbon (graphite phase) cluster size is around $12.9 \mathrm{~nm}$ for the deposit and 11.0 in the carbon halo with the graphitization induced by local irradiation of the electron beam ${ }^{37}$.

\section{CONCLUSIONS}

Electron beam induced deposition of silver from the gas phase could be achieved for the first time. By using $\mathrm{AgO}_{2} \mathrm{Me}_{2} \mathrm{Bu}$ as a precursor medium a silver content of about 73 at.\% could be realized by proper adjustment of the deposition parameters. While for high electron fluxes EBID deposits with a dominant carbon content were observed, small local fluxes pushed the growth of large silver crystals. Given the large amount of carbon in the precursor stoichiometry $(\mathrm{Ag}: \mathrm{C}=1: 6)$, this high silver content points to an effective desorption of the ligands under low electron irradiance. The suitability of such deposits for plasmonic applications was shown through surface 
enhanced Raman scattering of the carbon signal on planar deposits. One obvious next step would be integrating the corresponding GIS into a field emitting microscope in which a sufficient vertical growth rate allows for three-dimensional nanostructures. Due to the high local electron fluxes and resolution final silver nanostructures with an EBID core and silver mantle achieved through low dose post-irradiation can be envisaged. In conclusion, the presented study represents a key step for the fast and efficient direct writing of plasmonic silver nanostructures in combination with advanced local precursor chemistry realizing high-purity deposits.

\section{METHODS/EXPERIMENTAL}

Prior to deposition, precursor candidates were investigated concerning their atomic composition as well as their sensitivity against electron beam impact. One metal-organic precursor compound investigated was Ag-(2,2-dimethylbutanoato- $\kappa \mathrm{O})-$, CAS 1085717-13-0 ( $\left.\mathrm{AgO}_{2} \mathrm{Me}_{2} \mathrm{Bu}\right)$ consisting of cotton-like white filaments. $\mathrm{AgO}_{2} \mathrm{Me}_{2} \mathrm{Bu}$ showed a strong increase of the relative amount of silver in the precursor crystal filaments under electron beam exposure. This high sensitivity to electron beam impact in combination with the stability of the compound during rendered $\mathrm{AgO}_{2} \mathrm{Me}_{2} \mathrm{Bu}$ a promising candidate for electron beam induced deposition of silver (see Supporting Information, Supp1 and Supp2).

The deposition was carried out in a tungsten filament driven Hitachi S 3600 scanning electron microscope (SEM) equipped with a Xenos patterning engine and a home-built gas injection system (GIS). The evaporation unit of the GIS was optimized in terms of short and linear molecule paths to reduce temperature gradients and was built from chemically inert, stainless steel of type W.1.4445MV. Instead of the typical capillary an 'open-GIS' was preferred with a tube of $3 \mathrm{~mm}$ inner diameter since it provided for sufficiently large deposition rates especially for the low vapor 
pressure precursors used in this study (see Supporting Information, Supp5). For these technical reasons no valve could be integrated. The GIS system is fully introduced within the SEM chamber and mounted to a three-axis stage allowing for accurate positioning relative to the sample surface for the deposition. The stage and the GIS (evaporation) unit could be independently heated for the determination of the optimum deposition temperature windows. No carrier gas was used.

The systematic investigation of electron beam induced deposition began with the adjustment of the optimum temperature windows of both gas-injection system (GIS) and substrate. Therefore, spot depositions were performed using a $15 \mathrm{kV}$ acceleration voltage and $0.5 \mathrm{nA}(0.15 \mathrm{nA})$ beam current. The GIS was heated in steps of $10 \mathrm{~K}$ starting from $80^{\circ} \mathrm{C}$. Optimal deposition rates were reached for GIS temperatures of $150^{\circ} \mathrm{C}$. Experiments varying the substrate temperature showed that below $100^{\circ} \mathrm{C}$ condensation of the $\mathrm{AgO}_{2} \mathrm{Me}_{2} \mathrm{Bu}$ precursor sets in, while above ca. $126^{\circ} \mathrm{C}$ the selectivity of the process slowly decreases due to competing thermal dissociation (CVD) at the sample surface (see Supporting Information, Supp3) in vacuum. Both, condensation and CVD temperature define the substrate temperature window for selective electron beam induced deposition.

Microscope cover slips (BK7, $170 \mu \mathrm{m}$ ) with a transparent conductive coating of ITO (magnetron sputtered, $52.3 \mathrm{~nm}$ ), $\mathrm{n}$-doped silicon wafers and copper TEM grids with a carbon foil were used as substrates. Depositions on native oxide silicon and ITO-coated glass showed comparable results. While the glass substrates provide access to an optical characterization of the silver-based EBID deposits and guaranteed low thermal drifts of the heating stage, the silicon substrate served as a standard for Monte-Carlo modeling. Monte Carlo modeling was carried out using the open-source code Casino $^{38}$. 
For high-resolution imaging as well as for energy-dispersive X-ray spectroscopy (EDX) to determine the material composition a Hitachi S4800 equipped with an EDAX silicon drift detector (SDD) was employed. The used emission current of $10 \mu \mathrm{A}$ at an acceleration voltage of $8 \mathrm{kV}$ led to a sample current of $1 \mathrm{nA}$ at the substrate surface according to the optical settings. EDX analysis was performed with a take-off angle of $38^{\circ}$ during $100 \mathrm{~s}$ and the k-ratios of each atom were extracted from the silver deposit as well as the substrate. The background signal of the detector was subtracted from EDX spectra using EDAX TEAM ${ }^{\mathrm{TM}}$ software after the acquisition process. Atomic composition of the silver-based FEB deposits was calculated using the software SAMx STRATAGem for thin-film analysis. This allowed for the exclusion of the EDX signal contribution from the ITO layer and the glass substrate underneath the silver deposits. However, the carbon content due to co-deposition of the residual hydrocarbons during EDX signal acquisition still contributes to the overall composition. This carbon background is $15-16$ at.\% in the used system as quantified for an evaporated layer of pure silver on native oxide silicon (see Supporting Information, Supp 6). The topography of the deposits was monitored by atomic force microscopy (AFM) using a NT-MDT NTEGRA Spectra system. TEM lamellas were prepared using FEI Helios-600i focused ion beam (FIB) instrument, an approximately $20 \mathrm{~nm}$ gold layer was deposited onto the surface using sputtering, followed by an ion-beam induced deposition of a $\mathrm{Pt}$ protective layer. TEM and STEM images were acquired using a JOEL-2200FS microscope operated at $200 \mathrm{kV}$ acceleration voltage. Confocal Raman spectroscopy was performed using an upright ND-MDT NTEGRA Raman microscope featuring a laser source with a wavelength of $532 \mathrm{~nm}$ and a 100x objective lens with a numerical aperture of 0.95 . Spectra were recorded at a spectral resolution of $2.7 \mathrm{~cm}^{-1}$ with a $2 \mathrm{~s}$ exposure time for each point of the $15 \times 15 \mu \mathrm{m}^{2}$ Raman 
map with step size $200 \mathrm{~nm}$. The use of a neutral density filter maintained the laser power below $100 \mu \mathrm{W}$. 

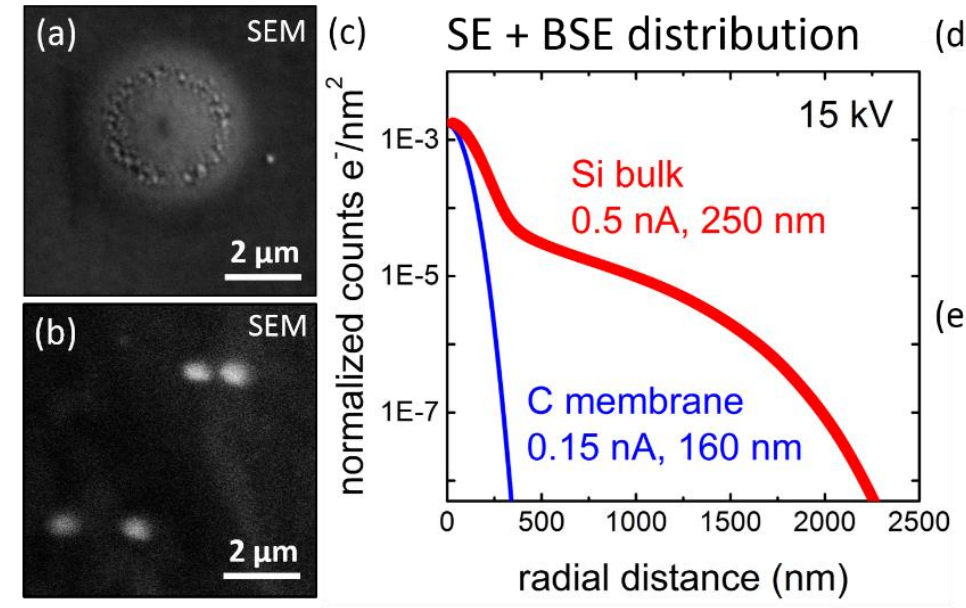

(d)

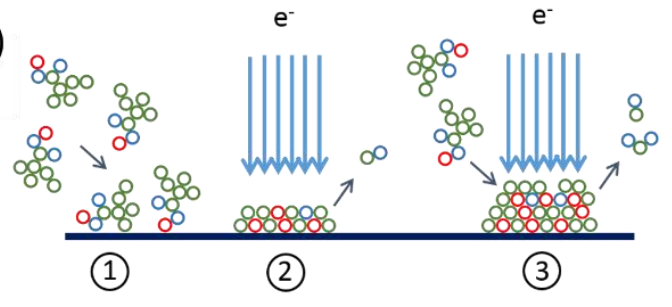

(e)

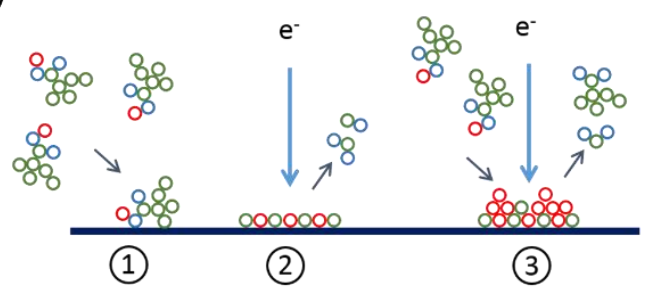

Figure 1. Scanning electron micrographs of spot deposits from $\mathrm{AgO}_{2} \mathrm{Me}_{2} \mathrm{Bu}$ : (a) typical spot deposit on bulk silicon, (b) spot deposits on TEM grid carbon membrane of $20 \mathrm{~nm}$ thickness, (c) Monte Carlo simulation of surface density distributions of secondary and backscattered electrons on bulk silicon (red) and carbon membrane (blue) generated by an electron beam of $15 \mathrm{keV}$. Beam current and FWHM are indicated. (d) and (e): Sketches of deposit formation processes for $\mathrm{AgO}_{2} \mathrm{Me}_{2} \mathrm{Bu}$, with red circles symbolizing the silver atoms while the colors signify elements of the organic ligand. Two different deposition regimes occur: (d) high electron flux: (1) physisorption, (2) electron beam induced co-dissociation of ligands leading to polymerization and surface poisoning, and (3) growth of carbon-rich deposit; (e) low electron flux: (1) physisorption, (2) electron beam induced dissociation and desorption of ligands, (3) growth of silver-rich deposits. 

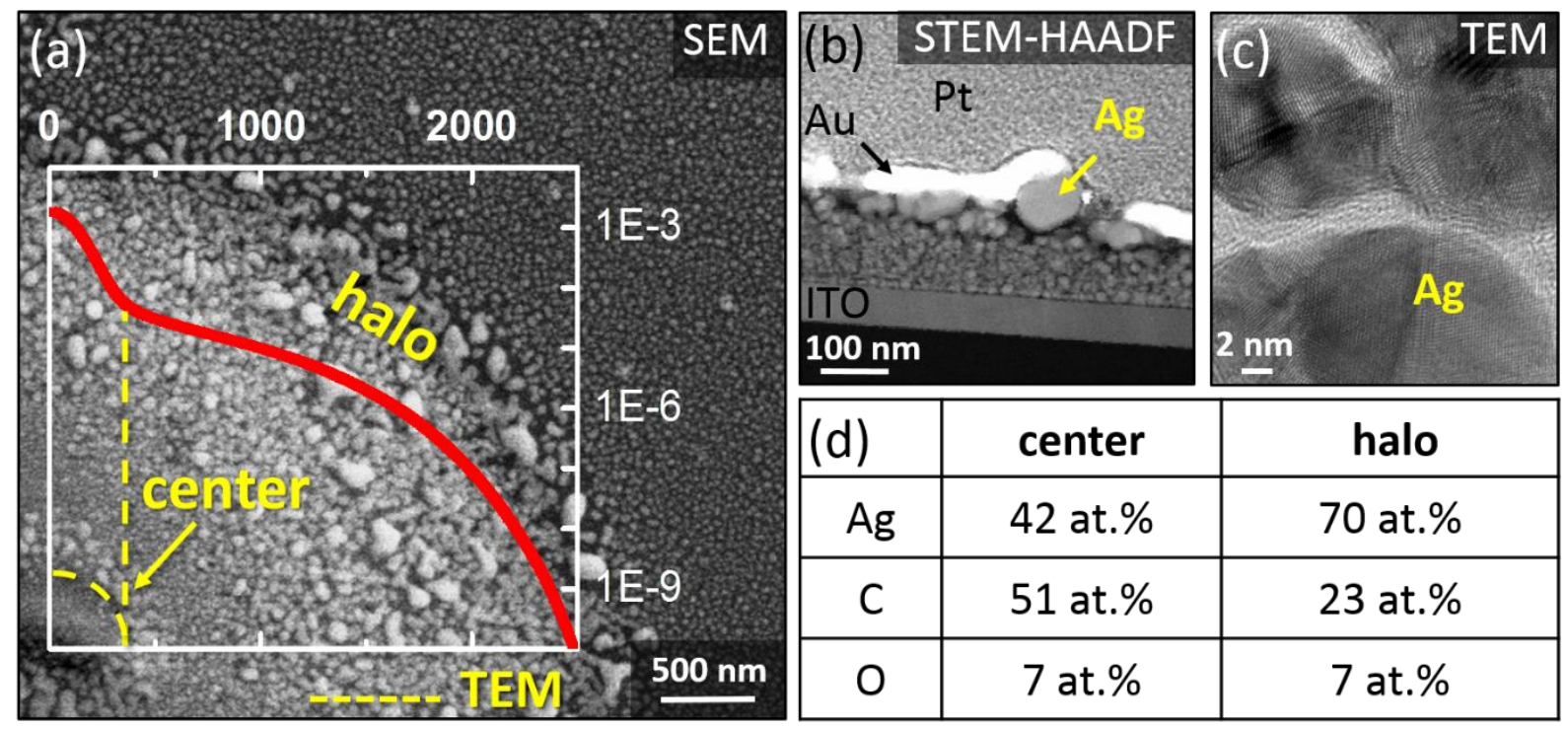

\begin{tabular}{|c|c|c|}
\hline$(d)$ & center & halo \\
\hline $\mathrm{Ag}$ & 42 at.\% & 70 at.\% \\
\hline $\mathrm{C}$ & 51 at.\% & 23 at.\% \\
\hline $\mathrm{O}$ & 7 at.\% & 7 at. $\%$ \\
\hline
\end{tabular}

Figure 2. Combinatorial approach to deposition regimes: (a) Scanning electron micrograph of a massive spot deposit from $\mathrm{AgO}_{2} \mathrm{Me}_{2} \mathrm{Bu}$ with (b) the corresponding cross-section shown in the high annular dark field scanning transmission electron micrograph (HAADF-TEM). (c) Transmission electron micrographs of the silver crystals in the transition region. The overlay in (a) shows the density of secondary and backscattered electrons from Fig. 1 (c) correlating with the regions for ligand co-dissociation in the central region (high electron density) and silver crystal growth in the halo (low electron density). (d) The table displays the thin-film and background corrected compositions. 

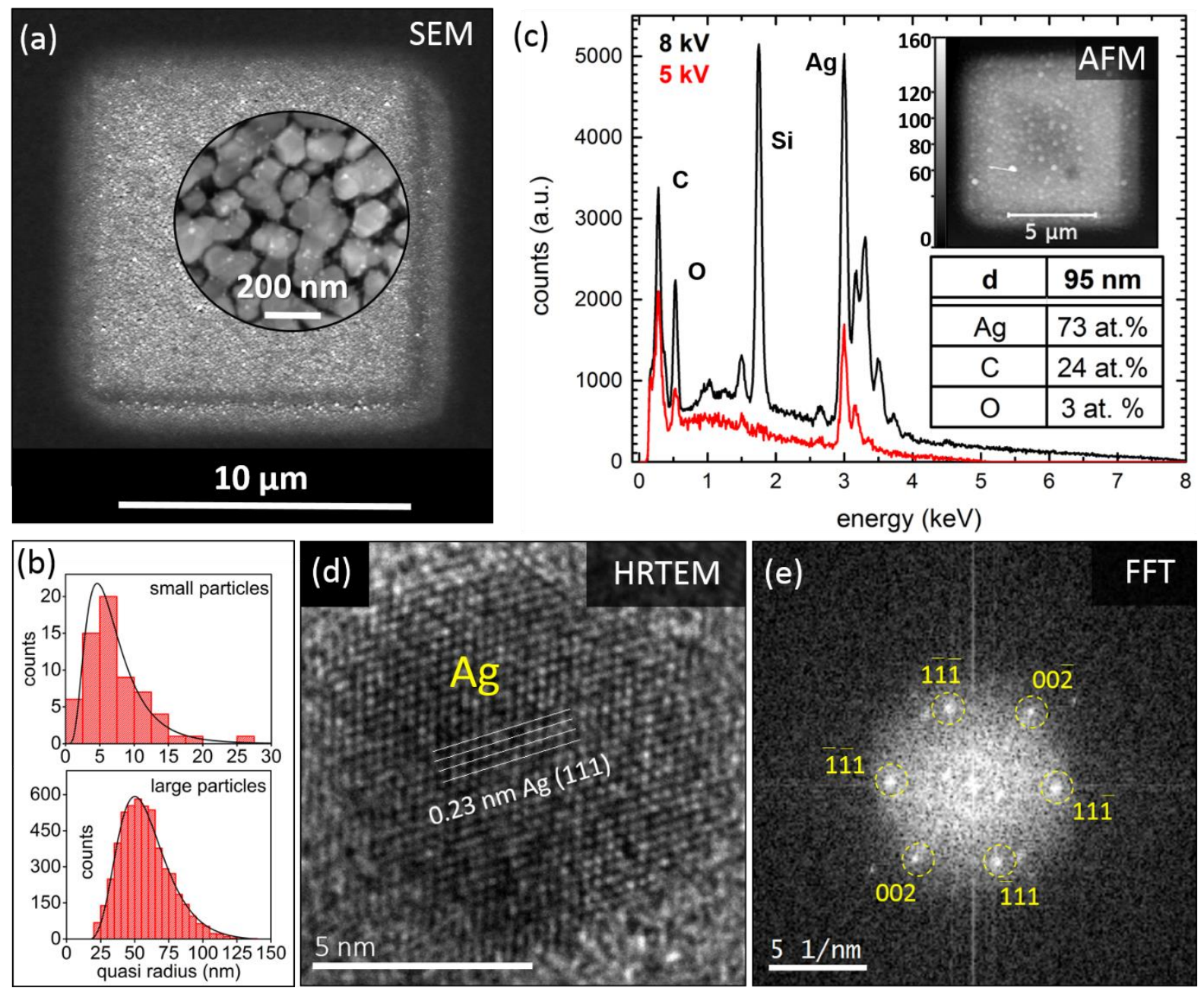

Figure 3. (a) Scanning electron micrograph of typical square deposit of $10 \times 10 \mu \mathrm{m}^{2}$ from $\mathrm{AgO}_{2} \mathrm{Me}_{2} \mathrm{Bu}$, (b) the histograms below show the particle size distributions in the deposits following a log-normal behavior, (c) EDX spectrum for two different incident energies, black: $8 \mathrm{keV}$, red: $5 \mathrm{keV}$, upper inset: AFM scan of the investigated deposit of thickness $\mathrm{d}=95 \mathrm{~nm}$, lower inset: material composition as obtained after thin-film correction, (d) HR-TEM image of silver crystal with the lattice distances measured for the (111) plane, (e) corresponding FFT denoting the diffraction spots for different lattice planes. 

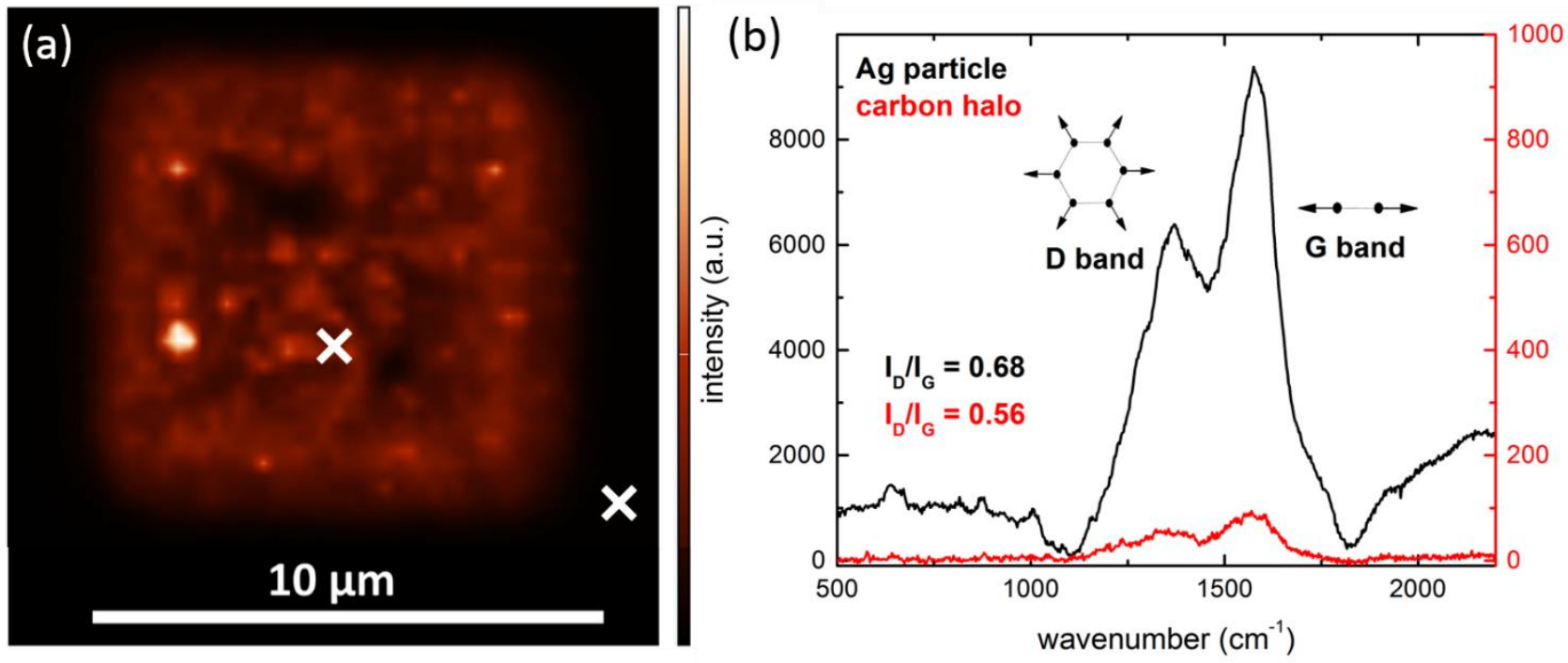

Figure 4. (a) 2D Raman intensity map of the silver pad shown in Figure 3, based on the maximum intensity of the collected spectra. The local signal enhancement from plasmonic excitation of the silver particles can be clearly distinguished. (b) Corresponding Raman spectra over the carbon vibration region for the two positions marked in (a). 


\section{ASSOCIATED CONTENT}

\section{Supporting Information.}

Supp1: Electron beam sensitivity and areas after irradiation

Supp2: Condensation tests prior to deposition

Supp3: Adjustment of stage temperature

Supp4: Beam profiles of the used electron beam

Supp5: Calculations of impinging gas flux for OpenGIS design

Supp6: Layer and background correction for systematic EDX investigation on

Supp7: TEM investigation of the deposits

Supp8: Size distributions of silver particles in the deposit

Supp9: Deposition tests for continued growth

\section{AUTHOR INFORMATION}

\section{Corresponding Author}

*katja.hoeflich@,helmholtz-berlin.de

\section{Author Contributions}

The manuscript was written through contributions of all authors. All authors have given approval to the final version of the manuscript.

\section{Funding Sources}

The research leading to this results was funded by the Helmholtz association within the Helmholtz Postdoc Programme under grant no. PD140. The authors furthermore gratefully 
acknowledge financial support by the EU COST action CM1301 'CELINA' (http://celina.unibremen.de/celina/).

\section{ACKNOWLEDGMENT}

The authors thank Oliver Feddersen Clausen from modularflow (http://www.modularflow.com/) for providing various precursor compounds and Anton Böll from Empa for his great job in the mechanical workshop.

\section{REFERENCES}

(1) Fowlkes, J. D. Simulation Guided 3D Nanomanufacturing via Focused Electron Beam Induced Deposition. ACS Nano 2016, 10, 6163-6172.

(2) Jesse, S.; Borisevich, A. Y.; Fowlkes, J. D.; Lupini, A. R.; Rack, P. D.; Unocic, R. R.; Sumpter, B. G.; Kalinin, S. V.; Belianinov, A.; Ovchinnikova, O. S. Directing Matter: Toward Atomic-Scale 3D Nanofabrication. ACS Nano 2016, 10 (6), 5600-5618.

(3) Utke, I.; Hoffmann, P.; Melngailis, J. Gas-Assisted Focused Electron Beam and Ion Beam Processing and Fabrication. J. Vac. Sci. Technol. B 2008, 26 (4), 1197-1276.

(4) Huth, M.; Porrati, F.; Schwalb, C.; Winhold, M.; Sachser, R.; Dukic, M.; Adams, J.; Fantner, G. Focused Electron Beam Induced Deposition: A Perspective. Beilstein J. Nanotechnol. 2012, 3, 597-619.

(5) Botman, A.; Mulders, J. J. L.; Hagen, C. W. Creating Pure Nanostructures from ElectronBeam-Induced Deposition Using Purification Techniques: A Technology Perspective. Nanotechnology 2009, 20 (37), 17pp. 
(6) Wnuk, J. D.; Rosenberg, S. G.; Gorham, J. M.; van Dorp, W. F.; Hagen, C. W.; Fairbrother, D. H. Electron Beam Deposition for Nanofabrication: Insights from Surface Science. Surf. Sci. 2011, 605 (3-4), 257-266.

(7) Spencer, J. A.; Rosenberg, S. G.; Wu, M. B. Y.; Fairbrother, L. M. D. H. Understanding the Electron-Stimulated Surface Reactions of Organometallic Complexes to Enable Design of Precursors for Electron Beam-Induced Deposition. Appl. Phys. A 2014, 117, 1631-1644.

(8) Schuller, J. a; Barnard, E. S.; Cai, W.; Jun, Y. C.; White, J. S.; Brongersma, M. L. Plasmonics for Extreme Light Concentration and Manipulation. Nat. Mater. 2010, 9 (3), 193-204.

(9) Höflich, K.; Becker, M.; Leuchs, G.; Christiansen, S. Plasmonic Dimer Antennas for Surface Enhanced Raman Scattering. Nanotechnology 2012, 23 (18), 185303.

(10) Winkler, R.; Schmidt, F. P.; Haselmann, U.; Fowlkes, J. D.; Lewis, B. B.; Kothleitner, G.; Rack, P. D.; Plank, H. Direct-Write 3D-Nanoprinting of Plasmonic Structures. ACS Appl. Mater. Interfaces 2016, 9, $8233-8240$.

(11) Brett B. Lewis, Michael G. Stanford, Jason D. Fowlkes, Kevin Lester, H. P. and P. D. R. Electron-Stimulated Purification of Platinum Nanostructures Grown via Focused Electron Beam Induced Deposition. Beilstein J. Nanotechnol. 2015, 6, 1883-1885.

(12) Belić, D.; Shawrav, M. M.; Gavagnin, M.; Stöger-Pollach, M.; Wanzenboeck, H. D.; Bertagnolli, E. Direct-Write Deposition and Focused-Electron-Beam-Induced Purification of Gold Nanostructures. ACS Appl. Mater. Interfaces 2015, 7 (4), 2467-2479.

(13) Geier, B.; Gspan, C.; Winkler, R.; Schmied, R.; Fowlkes, J. D.; Fitzek, H.; Rauch, S.; 
Rattenberger, J.; Rack, P. D.; Plank, H. Rapid and Highly Compact Purification for Focused Electron Beam Induced Deposits: A Low Temperature Approach Using Electron Stimulated H2O Reactions. J. Phys. Chem. C 2014, 118 (25), 14009-14016.

(14) Haverkamp, C.; Höflich, K.; Jäckle, S.; Manzoni, A.; Christiansen, S. Plasmonic Gold Helices for the Visible Range Fabricated by Oxygen Plasma Purification of Electron Beam Induced Deposits. Nanotechnology 2017, 28 (5), 55303.

(15) Stanford, M. G.; Lewis, B. B.; Noh, J. H.; Fowlkes, J. D.; Roberts, N. A.; Plank, H.; Rack, P. D. Purification of Nanoscale Electron-Beam-Induced Platinum Deposits via a Pulsed Laser-Induced Oxidation Reaction. ACS Appl. Mater. Interfaces 2014, 6 (23), 2125621263.

(16) Begun, E.; Dobrovolskiy, O. V; Kompaniiets, M.; Sachser, R.; Gspan, C.; Plank, H.; Huth, M. Post-Growth Purification of Co Nanostructures Prepared by Focused Electron Beam Induced Deposition. Nanotechnology 2015, 26 (7), 75301.

(17) Höflich, K.; Yang, R. Bin; Berger, A.; Leuchs, G.; Christiansen, S. The Direct Writing of Plasmonic Gold Nanostructures via Electron Beam Induced Deposition. Adv. Mater. 2011, 23 (22-23), 2657-2661.

(18) Puydinger dos Santos, M. V.; Velo, M. F.; Domingos, R. D.; Zhang, Y.; Maeder, X.; Guerra-Nuñez, C.; Best, J. P.; Béron, F.; Pirota, K. R.; Moshkalev, S.; Diniz, J. A.; Utke, I. Annealing-Based Electrical Tuning of Cobalt-Carbon Deposits Grown by FocusedElectron-Beam-Induced Deposition. ACS Appl. Mater. Interfaces 2016, 32496-32503.

(19) Utke, I.; Hoffmann, P.; Dwir, B.; Leifer, K.; Kapon, E.; Doppelt, P. Focused Electron Beam 
Induced Deposition of Gold. J. Vac. Sci. Technol. B 2000, 18 (6), 3168.

(20) Van Dorp, W. F.; Wu, X.; Mulders, J. J. L.; Harder, S.; Rudolf, P.; De Hosson, J. T. M. Gold Complexes for Focused-Electron-Beam-Induced Deposition. Langmuir 2014, 30 (40), $12097-12105$.

(21) Mulders, J. J. L.; Belova, L. M.; Riazanova, A.; Villamor, E.; Casanova, F. Fabrication of Pure Gold Nanostructures by Electron Beam Induced Deposition with Au ( $\mathrm{CO}$ ) $\mathrm{Cl}$ Precursor : Deposition Characteristics and Primary Beam. J. Phys. D Appl. Phys. 2012, 45, 475301.

(22) Walz, M.-M.; Schirmer, M.; Vollnhals, F.; Lukasczyk, T.; Steinrück, H.-P.; Marbach, H. Electrons As "invisible Ink": Fabrication of Nanostructures by Local Electron Beam Induced Activation of SiOx. Angew. Chemie 2010, 49 (27), 4669-4673.

EU COST Action CM1301 “CELINA.”

(24) Warneke, J.; Rohdenburg, M.; Zhang, Y.; Orzagh, J.; Vaz, A.; Utke, I.; De Hosson, J. T. M.; van Dorp, W. F.; Swiderek, P. Role of NH 3 in the Electron-Induced Reactions of Adsorbed and Solid Cisplatin. J. Phys. Chem. C 2016, 120 (7), 4112-4120.

(25) Wnuk, J. D.; Gorham, J. M.; Rosenberg, S. G.; van Dorp, W. F.; Madey, T. E.; Hagen, C. W.; Fairbrother, D. H. Electron Induced Surface Reactions of the Organometallic Precursor trimethyl(methylcyclopentadienyl)platinum(IV). J. Phys. Chem. C 2009, 113 (6), 24872496.

(26) Wnuk, J. D.; Gorham, J. M.; Rosenberg, S. G.; van Dorp, W. F.; Madey, T. E.; Hagen, C. W.; Fairbrother, D. H. Electron Beam Irradiation of Dimethyl-(Acetylacetonate) gold(III) 
Adsorbed onto Solid Substrates. J. Appl. Phys. 2010, 107 (5), 54301.

(27) Fisher, J. S.; Kottke, P. a.; Kim, S.; Fedorov, A. G. Rapid Electron Beam Writing of Topologically Complex 3D Nanostructures Using Liquid Phase Precursor. Nano Lett. 2015, 15 (12), 8385-8391.

(28) Ocola, L. E.; Joshi-Imre, A.; Kessel, C.; Chen, B.; Park, J.; Gosztola, D.; Divan, R. Growth Characterization of Electron-Beam-Induced Silver Deposition from Liquid Precursor. J. Vac. Sci. Technol. B Microelectron. Nanom. Struct. 2012, 30 (6), 06 FF08.

(29) Bresin, M.; Nehru, N.; Hastings, J. T. Focused Electron-Beam Induced Deposition of Plasmonic Nanostructures from Aqueous Solutions. Spie Moems-Mems 2013, 8613, 861306.

(30) Szczęsny, R.; Szłyk, E. Thermal Decomposition of Some silver(I) Carboxylates under Nitrogen Atmosphere. J. Therm. Anal. Calorim. 2013, 111 (2), 1325-1330.

(31) Szymanska, I.; Piszczek, P.; Szcz, R.; Szłyk, E. Thermal and MS Studies of Silver ( I ) 2 , 2-Dimethylbutyrate Complexes with Tertiary Phosphines and Their Application for CVD of Silver Films. Polyhedron 2007, 26, 2440-2448.

(32) van Dorp, W. F.; van Someren, B.; Hagen, C. W.; Kruit, P.; Crozier, P. a. Approaching the Resolution Limit of Nanometer-Scale Electron Beam-Induced Deposition. Nano Lett. 2005, $5(7), 1303-1307$.

(33) Silvis-Cividjian, N.; Hagen, C. W.; Kruit, P. Spatial Resolution Limits in Electron-BeamInduced Deposition. J. Appl. Phys. 2005, 98 (8), 84905.

(34) Stamplecoskie, K. G.; Scaiano, J. C.; Tiwari, V. S.; Anis, H. Optimal Size of Silver 
Nanoparticles for Surface-Enhanced Raman Spectroscopy. J. Phys. Chem. C 2011, 115 (5), $1403-1409$.

(35) Robertson, J. Diamond-like Amorphous Carbon. Mater. Sci. Eng. R 2002, 37 (4-6), 129281.

(36) Tuinstra, F.; Koenig, J. L. Characterization of Graphite Fiber Surfaces with Raman Spectroscopy. J. Compos. Mater. 1970, 4, 492-499.

(37) Kulkarni, D. D.; Rykaczewski, K.; Singamaneni, S.; Kim, S.; Fedorov, A. G.; Tsukruk, V. V. Thermally Induced Transformations of Amorphous Carbon Nanostructures Fabricated by Electron Beam Induced Deposition. ACS Appl. Mater. Interfaces 2011, 3 (3), 710-720.

(38) CASINO - Monte CArlo SImulation of electroN Trajectories in sOlids. 
TOC FIGURE

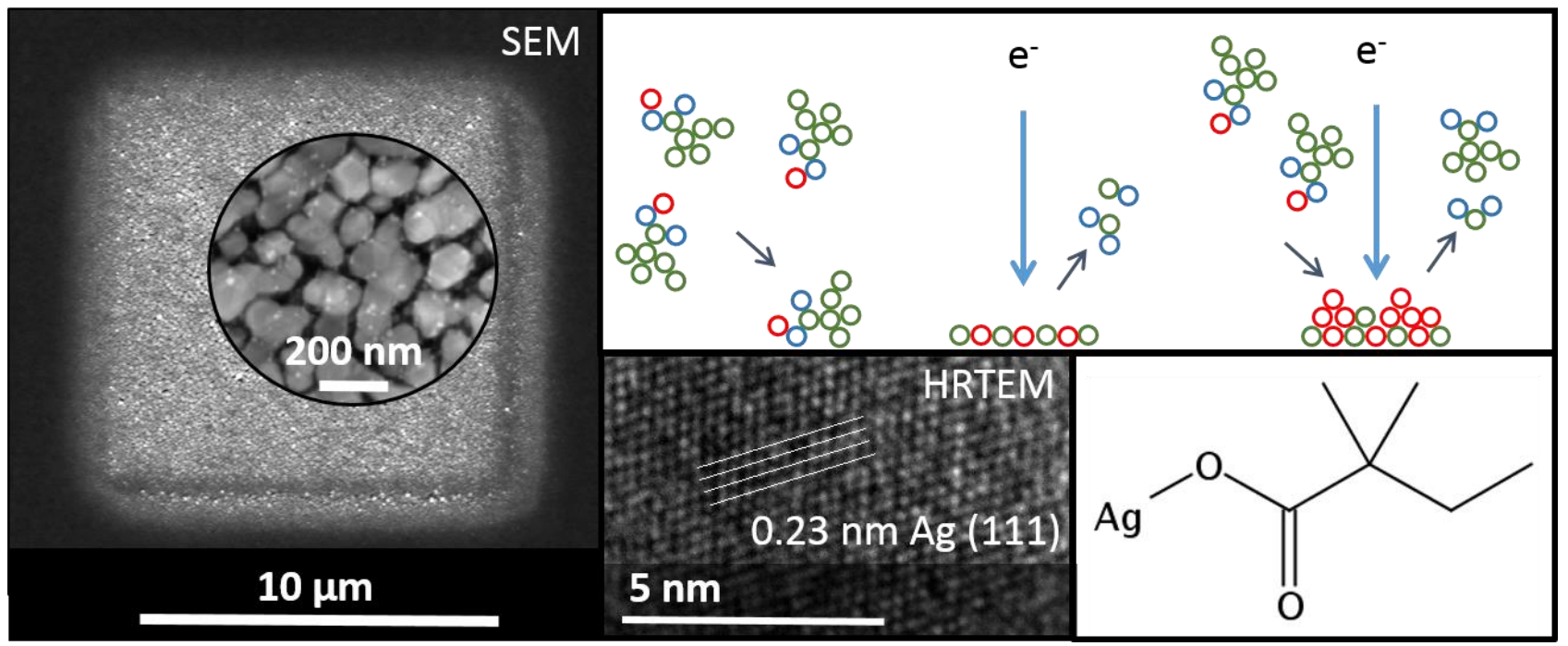

